\title{
AV Block Second Degree Mobitz Type I
}

National Cancer Institute

\section{Source}

National Cancer Institute. AV Block Second Degree Mobitz Type I. NCI Thesaurus. Code C62017.

A disorder characterized by an electrocardiographic finding of intermittent failure of atrial electrical impulse conduction to the ventricles, characterized by a progressively lengthening PR interval prior to the block of an atrial impulse. (CDISC) 